\title{
Bronchiolitis - It Is Time for a Unique Definition
}

\author{
Fabio Midulla and Raffaella Nenna
}

Department of Paediatrics and Infantile Neuropsychiatry, 'Sapienza' University of Rome, Rome, Italy

DOl: http://doi.org/10.17925/ERPD.2016.02.01.12

\begin{abstract}
Bronchiolitis is the most common lower respiratory tract infections in infants. It is time to reach a unique clinical definition, encompassing the acute onset of respiratory distress with cough, tachypnoea, retraction and diffuse crackles on auscultation in infants aged less than 12 months.
\end{abstract}

\section{Keywords}

Bronchiolitis, definition, age, respiratory syncytial virus

\begin{abstract}
Disclosure: Fabio Midulla and Raffaella Nenna have nothing to declare in relation to this article. No funding was received for the publication of this paper. This article is a short opinion piece and has not been submitted to external peer reviewers.

Open Access: This article is published under the Creative Commons Attribution Noncommercial License, which permits any non-commercial use, distribution, adaptation and reproduction provided the original author(s) and source are given appropriate credit.

Received: 20 January 2016 Published Online: 3 May 2016 Citation: European Respiratory \& Pulmonary Diseases, 2016;2(1):12-3

Correspondence: Fabio Midulla, Department of Paediatrics, 'Sapienza' University of Rome,V le Regina Elena 324, 00161, Rome, Italy. E: midulla@uniroma1.it
\end{abstract}

Acute bronchiolitis is the most common lower respiratory tract infection in infants less than 12 months of age, and it is the leading cause of hospitalisation in this age group, particularly in infants younger than six months. It is mainly caused by respiratory syncytial virus (RSV) and shows a seasonal pattern, with peak incidence occurring during winter, but other respiratory viruses can often be involved. ${ }^{1}$ Particularly, infants with RSV bronchiolitis are younger, have been breastfed for a shorter time and have a more severe form of bronchiolitis with prevalent chest X-ray findings of diffuse air trapping, whereas, human Rhinovirus (hRV), the second most frequent virus involved, seems to cause a milder form of bronchiolitis and affecting infants with a higher eosinophils count and, possibly, with an atopic predisposition. ${ }^{1}$ Each year 150 million new cases of bronchiolitis are reported worldwide with an increasing trend in medical visits and total hospitalisations along with the diagnosis of bronchiolitis.

Despite the fact that bronchiolitis is an 'old' disease, several aspects are still debated. First, a clear definition of this entity is still lacking. According to the European definition, bronchiolitis is a clinical diagnosis requiring epidemiological data, such as the epidemic period (December to March), the age of infants and the specific clinical appearance (signs of an upper respiratory infection: runny nose, fever, chough, followed by respiratory distress with diffuse crackles on auscultation). ${ }^{2}$ On the contrary, the American Pediatric Academy defines bronchiolitis as the first episode of acute viral wheeze occurring in infants less than two years old. It is evident that this definition likely overlaps the early presentation of wheezing or bronchial hyper-responsiveness. ${ }^{3}$ To exclude infants with virus-associated episodes of wheezing, we should consider bronchiolitis only in infants younger than 12 months presenting with their first episode of lower respiratory infection, who had diffuse crackles on auscultation. ${ }^{4}$ Wheezing is a high-pitched, musical, adventitious lung sound produced by turbulent airflow through a narrowed airway, whereas crackles are caused by the 'popping open' of small airways and alveoli collapsed by fluid, thus possibly reflecting two different entities affecting two different lung structures.
Second, it is a self-limiting disease and it should be managed only with supportive care. Reduced feeding is common and very young infants may present with apnoeic episodes, thus leading to hospitalisation, which is required by $2-3 \%$ of infants affected. ${ }^{5}$ Some patients, particularly when risk factors are present, can manifest a severe disease and the admission to the intensive care unit may be required. Few predisposing factors may explain the clinical worsening, leading to paediatric intensive care unit admission for ventilatory support. Young age (under one month), possibly due to the small size of the airways, and RSV carriage are the most important demographic factors associated with severe respiratory distress that is uncommon in previously healthy term infants and often develops soon after disease onset.

Furthermore, the conflict that exists in the definition creates many problems, especially in the interpretation of results of clinical trials and the relationship between bronchiolitis and wheezing and asthma. A single definition of bronchiolitis will allow an analysis of the homogeneous disease pathogenesis and the possible role of new therapeutic strategies. The exclusive use of supportive treatments (nasal washing, $\mathrm{O}_{2}$ and hydration) is restricted for pure bronchiolitis infants. In fact, bubbly nasopharyngeal secretions, by obstructing the nostrils may cause transient decreases in $\mathrm{SpO}_{2}$ in infants that are obligate nasal breathers. On the other hand, several medical therapies might be useful in wheezing children 12-24 months old. For example, the use of bronchodilators, as well as of corticosteroids, is no longer recommended for bronchiolitis infants, but may play a role in wheezing children. Overlapping these two diagnoses could result in erroneous management of children with respiratory failure. Nowadays, there is a huge variability in the clinical management of this disease around the world, including widespread use of unnecessary tests and ineffective therapies.

Moreover, long-standing evidence underlines the role of bronchiolitis in the occurrence of subsequent wheezing and asthma, especially in infants with hRV infection and a high eosinophils count during the 
disease. ${ }^{6}$ The use of asthma controller medication in children after hospitalisation for bronchiolitis is equally high with increasing health costs. Host and viral factors actively interact during respiratory infections, but it is still debated whether the virus directly modifies the host immune response or it simply identifies infants somehow prone to wheeze.

Recent evidence suggests that the age limit should be further restricted to six months because the clinical entity seems to even differ over this age in terms of aetiology, clinical severity and possibly pathogenesis. ${ }^{4}$ It remains to be determined whether the clinical manifestations of bronchiolitis differ in children younger than six months or if it is a separate disease.

In conclusion, we believe that bronchiolitis needs to reach a unique definition, characterised by acute onset of respiratory distress with cough, tachypnoea, retraction and diffuse crackles on auscultation in infants less than 12 months, or even six months of age.

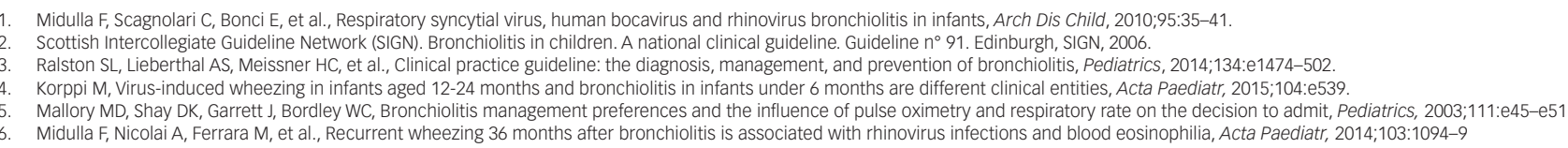

